\title{
Indoleamine 2,3-dioxygenase expression is associated with chronic rhinosinusitis with nasal polyps and antrochoanal polyps*
}

\author{
Teemu Honkanen ${ }^{1,2}$, Annika Luukkainen ${ }^{3}$, Mikko Lehtonen ${ }^{3,4}$, Timo \\ Paavonen $^{1,2}$, Jussi Karjalainen ${ }^{5}$, Mikko Hurme ${ }^{6,7}$, Jyri Myller $^{3,8}$, Heini \\ Huhtala $^{9}$, Markus Rautiainen ${ }^{3,4}$, Sanna Toppila-Salmi ${ }^{3,10,11}$
1 Department of Pathology, University of Tampere, Tampere, Finland
2 Department of Pathology, Centre for Laboratory Medicine, Tampere University Hospital, Tampere, Finland
3 Department of Clinical Medicine, University of Tampere, Tampere, Finland
4 Department of Eye, Ear and Oral diseases, Tampere University Hospital, Tampere, Finland
5 Allergy Centre, Tampere University Hospital, Tampere, Finland
6 Department of Microbiology, University of Tampere, Tampere, Finland
7 Department of Microbiology, Centre for Laboratory Medicine, Tampere University Hospital, Tampere, Finland
8 Department of Otorhinolaryngology, Paijat-Hame Central Hospital, Lahti, Finland
9 School of Public Health, University of Tampere, Tampere, Finland
${ }^{10}$ Transplantation laboratory, Haartman Institute, University of Helsinki, Helsinki, Finland
${ }^{11}$ Helsinki University Central Hospital, Skin and Allergy Hospital, Helsinki, Finland

SUMMARY

\begin{abstract}
Chronic rhinosinusitis without and with nasal polyps (CRSwNP and CRSSNP), and antrochoanal polyps are different phenotypes with different pathomechanisms. Indoleamine 2,3-dioxygenase (IDO) is an enzyme expressed in many cells involved in the catabolism of the essential amino acid tryptophan to kynurenine. IDO might have a role in allergic airway inflammation. The aim was to evaluate if IDO expression is associated with CRSSNP, $C R S w N P$, or ACP. One hundred fifty specimens from the nasal cavity and sinus mucosa were immunohistochemically stained with $m A b$ anti-IDO. The expression of epithelial and leukocyte IDO was associated with CRSwNP and ACP. The presence of ASA intolerance, asthma, atopy, smoking and use of medication did not significantly change the results. The different expression of IDO could putatively indicate the differences in the pathomechanisms of CRSSNP, CRSwNP and ACP. Further studies on the role of IDO in upper airways pathologies are required.
\end{abstract}

Key words: aspirin intolerance, chronic rhinosinusitis, eosinophil, indoleamine 2,3 dioxygenase, nasal polyp

\section{INTRODUCTION}

Chronic rhinosinusitis (CRS) is a heterogeneous group of inflammatory diseases of the nose and paranasal sinuses lasting for at least 12 weeks without resolution ${ }^{(1)}$. It is a significant health problem with a prevalence of $10-16 \%{ }^{(1)}$. It is frequently associated with asthma but their inter-relationship is poorly understood ${ }^{(1,2)}$. Hence, the global research goal is to acquire knowledge on patient phenotypes, subtypes, and the factors amplifying mucosal inflammation in CRS ${ }^{(3)}$. CRS with nasal polyps (CRSwNP) and without (CRSsNP) are considered to be phenotypes of CRS with possibly different aetiologies and pathomechanisms, although they may also be interpreted as different degrees of inflammation ${ }^{(3,4)}$. Nasal polyps (NP) appear as oedematous masses originating from the middle meatus and affect between 1 and $4 \%$ of the general population (1). Hyperplasic CRS has been an ill-defined group, representing putatively an overlap between CRS and NP ${ }^{(1)}$. Although CRS with hypertrophic polypoid sinus mucosa (HP) is also

Footnote: Honkanen \& Luukkainen contributed equally to this manuscript

Abbreviations: ACP = antrochoanal polyp; ASA = acetylic salicylic acid, aspirin; CRS = chronic rhinosinusitis; CRSsNP = chronic rhinosinusitis without nasal polyps; CRSwNP = chronic rhinosinusitis with nasal polyps; HP = hypertrophic polypoid sinus mucosa; IDO = Indoleamine 2,3-dioxygenase; Ig = immunoglobulin ; NP = nasal polyp; Th1 = T-helper cell 1; Th2 = T-helper cell 2 
lacking clinical characterization, its histological characters seem to be tortuous edematous mucosa with a putatively non increased number of glands, vessels, or eosinophils ${ }^{(5)}$.

Sinus specimens obtained from patients suffering from CRSsNP are generally characterised by basement membrane thickening, goblet cell hyperplasia, subepithelial oedema, abundant mononuclear cells and few eosinophils ${ }^{(1,6)}$. Histomorphological characterisation of CRSwNP reveals frequent epithelial damage, a thickened basement membrane, oedematous to sometimes fibrotic stromal tissue, with a reduced number of vessels and glands, but virtually no neural structure ${ }^{(7)}$. Eosinophil numbers are significantly higher in polyp tissue compared to CRSsNP ${ }^{(4)}$. CRSsNP is characterized by a T-helper cell 1 (Th1) polarization with high levels of interferon-gamma (IFN- $\gamma$ ) and transforming growth factor beta (TGF- $\beta$ ), while CRSwNP is characterised by a T-helper cell 2 (Th2) polarization with high interleukin 5 (IL-5) and immunoglobulin E (IgE) concentrations ${ }^{(8)}$. A deficit in $\mathrm{T}$ regulatory cell capacity in CRSwNP might lead to a strong increase in Th1 and Th2 effector cell signals ${ }^{(9)}$. Antrochoanal polyps (ACP) originate (unilaterally) from the maxillary sinus mucosa and protrude into the choana. Although macroscopically similar to the classic NP, ACP is considered to have a predominance of neutrophils ${ }^{(10)}$.

In patients with aspirin (ASA) sensitivity, 36-96\% have CRSwNP ${ }^{(11,12)}$. CRSwNP in ASA hypersensitive patients is characterised by involvement of all sinuses and nasal passages and thicker hypertrophic mucosa with abundant eosinophils (13). Although the pathogenesis of chronic eosinophilic inflammation of the airway mucosa and nasal polyps in ASAsensitive patients does not seem to be related to intake of aspirin or other nonsteroidal anti-inflammatory drugs, it has been speculated that the pathomechanism underlying CRSwNP in ASA sensitive patients may be different from that in ASA tolerant patients ${ }^{(1)}$.

Indoleamine 2,3 dioxygenase (IDO) is an intracellular enzyme that initiates the first and rate-limiting step of tryptophan breakdown along the kynurenine pathway ${ }^{(14)}$. IDO is widely expressed in a variety of cell types including leukocytes and tumour cells ${ }^{(15)}$. Initially the role of IDO was thought to be mainly antimicrobial by reducing the availability of the essential amino acid tryptophan in the inflammatory environment ${ }^{(16)}$. In the past years, IDO has emerged as an important regulator of the immune system; however, it is not known whether local IDO activity is beneficial or detrimental to inflamed tissues. IDO is induced by IFN- $\gamma$ and other inflammatory cytokines during inflammation or as a consequence of normal tissue function ${ }^{(17)}$. IDO suppresses $\mathrm{T}$ cell activity and promotes $\mathrm{T}$ cell tolerance to further antigenic challenges, by promoting the differentiation of naïve CD4 $\mathrm{T}$ cells into regulatory $\mathrm{T}$ cells, regulated putatively by dendritic cells ${ }^{(18-22)}$. IDO seems to serve as a negative feedback loop or is not essential for Th1 response, but it plays a distinct role in up-regulating
Th2 dominant immune responses ${ }^{(15,23)}$. Moreover, IDO has also been shown to down-regulate Th2 responses ${ }^{(24)}$. The role of IDO in the modulation of allergic airway inflammation has recently been investigated ${ }^{(25-28)}$.

As there may be pathophysiological differences between CRSsNP, CRSwNP, and ACP, our objective was to compare the sinonasal epithelial and mucosal expression of IDO in these subgroups.

\section{MATERIALS AND METHODS}

Subjects

This study was carried out at the Department of Otorhinolaryngology, Tampere University Hospital, Finland and has been approved by the Hospital's Ethical committee. All the subjects were Caucasian. Subject groups are shown in Tables 1 and 2. The inclusion criteria of patients were: diagnosis of CRSsNP, CRSwNP, or ACP based on EPOS criteria of symptoms, endoscopic and sinus computed tomography findings ${ }^{(1)}$. The exclusion criteria were cystic fibrosis, and diseases with a severe impact on general immunity. The exclusion criteria of control subjects were: sinonasal disease (except mild allergic rhinitis), or any other disease requiring constant medication. Diagnosis of atopy was based on skin prick test positivity. Diagnosis of asthma was based on clinical features and pulmonary function tests. Diagnosis of ASA intolerance was made on the basis of a history of wheezing or asthma attacks precipitated by non-steroidal anti-inflammatory drugs.

The first set of specimens was taken from the nasal cavity. Control nasal mucosa was obtained from the inferior turbinate under local anaesthesia from 19 healthy, non-atopic volunteers who did not use any medication. Polyp specimens were obtained from patients for diagnostic purposes or undergoing endoscopic polypectomy with or without sinus surgery, under local or general anaesthesia. The maxillary sinus specimens were taken from different individuals than the specimens of the first set. Control sinus mucosa was obtained from 12 subjects under general anaesthesia during bimaxillary osteotomy. Maxillary sinus specimens from patients with CRS were obtained during endoscopic sinus surgery under local or general anaesthesia.

\section{Tissue handling}

Nasal specimens, obtained from healthy volunteers, were immediately snap-frozen in liquid nitrogen and stored at $-80^{\circ} \mathrm{C}$ until analysis. Other specimens, obtained at the time of surgery, were formalin-fixed and paraffin-embedded.

\section{Sample staining}

Tissue samples were stained with hemalaun-eosin for calculating the number of mucosal eosinophils $/ \mathrm{mm}^{2}$. The polyp specimens were additionally stained with Periodic acid-Schiff (PAS) for evaluating semiquantitatively the amount of mucus on the polyp surface (PAS $+=$ little or no mucus, PAS $++=$ much mucus). 


\section{Immunohistochemistry}

For light microscopic evaluation, specimens were stained with immunoperoxidase staining, 3-5 $\mu \mathrm{m}$ thick frozen or paraffin sections were cut onto Superfrost Plus microscope slides (Menzel-Gläser, Braunschweig, Germany). Frozen sections were fixed with formalin for 45 minutes. Fully automated immunostaining was performed by Ventana BenchMark LT Automated IHC Stainer (Ventana Medical System, Arizona, USA). Ultraview Universal DAB detection kit (catalogue No. 760-500, Ventana Medical System, Arizona, USA) was used. Ventana EZ Prep solution (catalogue No 950-100, Ventana) was used for deparaffinisation. For epitope retrieval CC1:
Tris-EDTA buffer pH 8.0 (catalogue No 950-124,Ventana) was used at $95^{\circ} \mathrm{C}$ to $100^{\circ} \mathrm{C}$ for 30 minutes with paraffin embedded tissue sections, and 8 minutes with frozen sections. Endogenous peroxidase was blocked with UV-Inhibitor 3\% $\mathrm{H}_{2} \mathrm{O}_{2}$ (Ventana) for 4 minutes at $37^{\circ} \mathrm{C}$. Tissue slides were rinsed between steps with Ventana Tris-based Reaction buffer (catalogue No. 950-300, Ventana). Slides were incubated at $37^{\circ} \mathrm{C}$ for 32 minutes with $\mathrm{mAb}$ anti-Indoleamine 2,3 dioxygenase (1:200, clone MAB5412, Chemicon International Inc., USA) followed by application of Ventana Ultraview HRP Universal Multimer $\left(8\right.$ minutes at $\left.37^{\circ} \mathrm{C}\right)$. Diaminobenzidine (DAB) was used as a chromogen and haematoxylin as a nuclear stain.
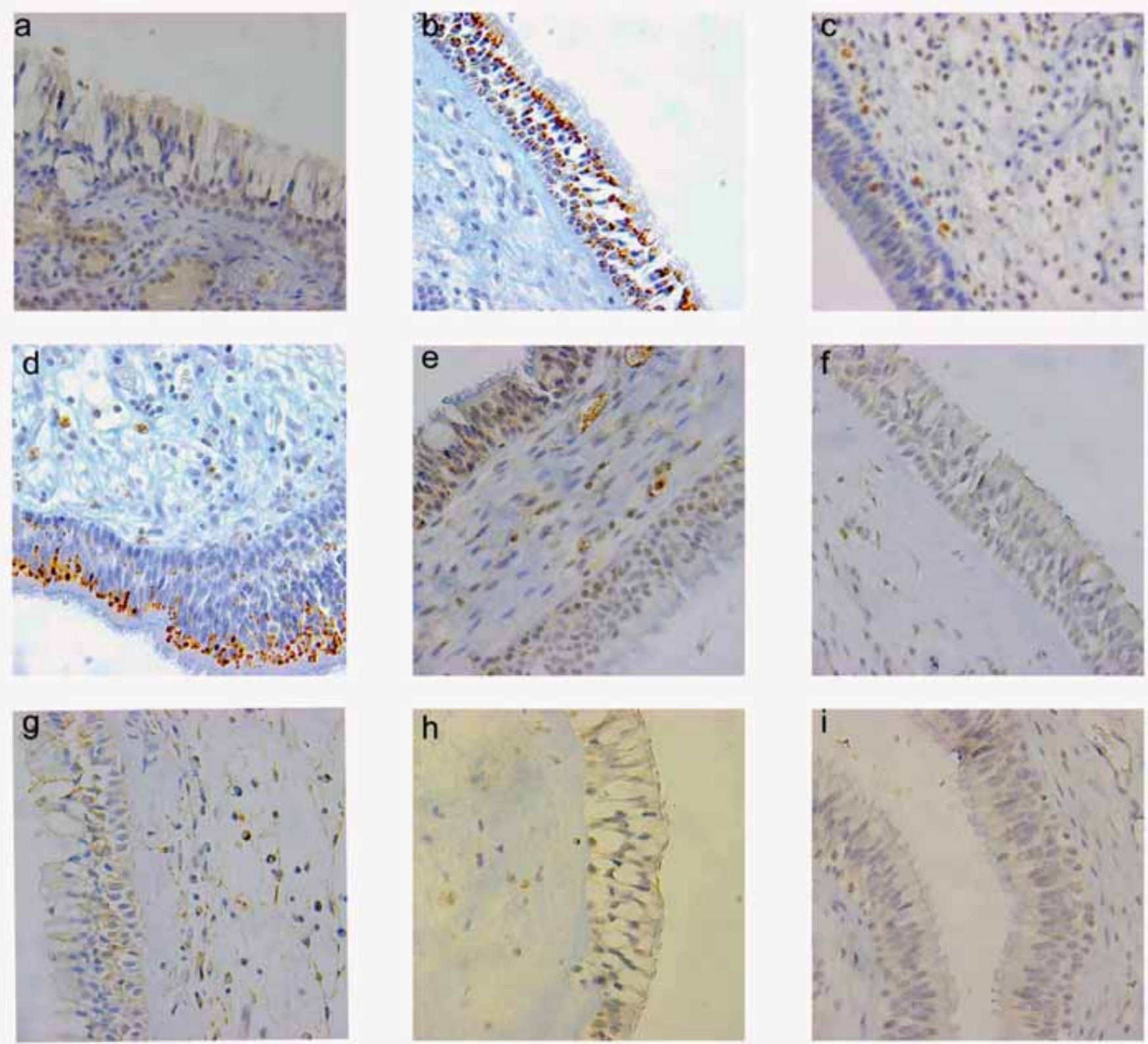

Figure 1. The expression of indoleamine 2,3-dioxygenase (IDO) in biopsies from the nasal cavity (a-d) and maxillary sinus mucosa (e-i). (a) Control nasal mucosa without epithelial or leukocyte expression of IDO. (b) Chronic rhinosinusitis with nasal polyp (CRSwNP) with epithelial IDO+ and leukocyte IDO-. (c) ASA-intolerant CRSwNP with epithelial IDO- and leukocyte IDO+. (d) Antrochoanal polyp with epithelial IDO+ and leukocyte IDO+. (e) Control sinus mucosa with epithelial IDO- and leukocyte IDO+. (f) CRSsNP with epithelial IDO- and leukocyte IDO-. (g) CRSsNP with epithelial IDO- and leukocyte IDO+. (h) CRSwNP with epithelial IDO- and leukocyte IDO+. (i) ASA-intolerant CRSwNP with epithelial IDO- and leukocyte IDO-. Original magnification 400x. 
Known positive tissue samples (from coeliac or inflammatory bowel disease) were also used to confirm the staining reliability of all separate staining patches ${ }^{(29,30)}$. The specificity of immunohistochemistry was controlled by omitting the primary antibodies or replacing them with irrelevant antisera.

\section{Light microscopic evaluation}

Sections were examined with a Leica DM 2000 light microscope (Leica Microsystems GmbH, Wetzlar, Germany) by two independent observers blinded to the experimental conditions. In a sample, there was either moderate or strong immunoreactivity of all epithelial cells or no epithelial positivity at all. Thus, the results were expressed as IDO positive epithelium or IDO negative epithelium. The percentage of IDO positive leukocytes was assessed. The number of eosinophils $/ \mathrm{mm}^{2}$ was counted from Hemalaun-Eosin staining. Diagnosis of hypertrophic polypoid mucosa (HP) of the maxillary sinus was based on microscopic evaluation of Hemalaun-Eosin stained sinus specimens by two blinded observers (T.P. and S.T-S). HP was semiquantitatively graded in specimens from patients with CRS: HP- = no or mild tortuous edematous mucosa, mild epithelial damage; CRS HP+ = moderate or severe tortuous edematous mucosa, moderate to severe epithelial damage.

\section{Data analysis}

Statistical analysis was carried out by the SPSS Base 15.0 Statistical Software Package (SPSS Inc., Chicago, IL, USA). Data is expressed as medians or means. For comparisons, the results were analysed by, Fisher's exact, Kruskal-Wallis, Mann Whitney U tests, and binary logistic regression analysis. Two-tailed p-values of $<0.05$ were considered statistically significant.

\section{RESULTS}

Expression of IDO in the nasal cavity: control inferior turbinate, $C R S w N P$, and $A C P$

IDO was strongly expressed in the vicinity of the Golgi apparatus of epithelial cells, but not on the supraepithelial mucus (Figure 1). IDO was additionally expressed weakly in submucosal leukocytes and intraepithelial glands (Figure 1).

Compared to control inferior turbinate, epithelial IDO+ was significantly associated with CRSwNP and ACP ( $p<0.01$, by logistic regression analysis, Table 3 ). The findings remained the same, when adjusted by aspirin-intolerance, atopy, asthma, smoking, use of intranasal or peroral corticosteroids, or antihistamines, previous operations, recurrence of polyps, sex and age $(\mathrm{p}<0.05$, by logistic regression analysis, data not shown).

Compared to control inferior turbinate, the percentage of IDO positive leukocytes was significantly higher in CRSwNP and ACP specimens ( $p<0.001, p<0.05$, respectively, by KruskalWallis and Mann Whitney U test, Table 1). There were no differences in the median percentage of IDO positive leukocytes between the existence or not of the following factors: aspirinintolerance, atopy, asthma, smoking, use of intranasal or peroral corticosteroids, or antihistamines, previous operations, recurrence of polyps, and sex ( $\mathrm{p}>0.05$ by Kruskal-Wallis test, data not shown). Age did not correlate with the percentage of IDO positive leukocytes ( $p>0.05$ by Spearman rank correlation test, data not shown). The median percentage of IDO positive leukocytes was higher in specimens with epithelial IDO+ than that with epithelial IDO- (median percentages of IDO positive eosinophils $13 \%$ and $0 \%$, respectively, $\mathrm{p}<0.001$ by Kruskal-Wallis and Mann Whitney U tests, data not shown).

The median number of mucosal eosinophils was higher in CRSwNP and ACP specimens compared to control inferior turbinate $(\mathrm{p}<0.001$ by Kruskal-Wallis and Mann Whitney $\mathrm{U}$ test, Table 1). The median number of eosinophils was significantly higher in epithelial IDO+ than in epithelial IDO- groups (median numbers of mucosal eosinophils $110.4 / \mathrm{mm}^{2}$ and $0.04 /$ $\mathrm{mm}^{2}$, respectively, $\mathrm{p}<0.001$ by Kruskal-Wallis and Mann Whitney U test, data not shown). Moreover, the number of eosinophils $/ \mathrm{mm}^{2}$ correlated significantly with the percentage of IDO + leukocytes $(p<0.01, r=0.41$, by Spearman rank correlation test, data not shown).

The epithelial IDO+ was associated with high amount of supraepithelial mucus, e.g. strong PAS positivity ( $p<0.05$, by Fisher's exact test, data not shown). A higher number of eosinophils was also significantly associated with the high amount of supraepithelial mucus (median numbers of mucosal eosinophils $171.2 / \mathrm{mm} 2$ and $65.6 / \mathrm{mm} 2$ in PAS++ and PAS+ groups respectively, $\mathrm{p}<0.05$ by Kruskal-Wallis and Mann Whitney $\mathrm{U}$ test, data not shown). In contrast, the median percentage of IDO positive leukocytes did not significantly associate with the amount of supraepithelial mucus ( $p>0.05$ by KruskalWallis test, data not shown).

Expression of IDO in the maxillary sinus mucosa: control, CRSsNP, and CRSwNP

IDO was expressed in the sinus epithelium and submucosal leukocytes in a similar way as in the specimens taken from the nasal cavity (Figure 1).

Compared to control sinus mucosa, the epithelial IDO+ did not statistically differ in CRSsNP or CRSwNP groups ( $p>$ 0.05 , by Fisher's exact test, Table 2, and by logistic regression test, data not shown). These findings remained insignificant when adjusted to aspirin intolerance, asthma, atopy, smoking, previous operations, microscopic evidence of hypertrophic polypoid sinus mucosa, use of intranasal or peroral corticosteroids, sex, and age ( $\mathrm{p}>0.05$, by logistic regression analyses, data not shown).

In contrast, the median percentage of IDO positive leukocytes was significantly higher in CRSwNP specimens compared to both control sinus mucosa and CRSsNP groups ( $p$ $<0.05$, by Kruskal-Wallis and Mann Whitney U test, Table 2). There were no differences in the percentage of IDO positive leukocytes between the existence or not of the following factors: aspirin-intolerance, atopy, asthma, smoking, use of 
Table 1. Patient characteristics and results of specimens taken from the nasal cavity.

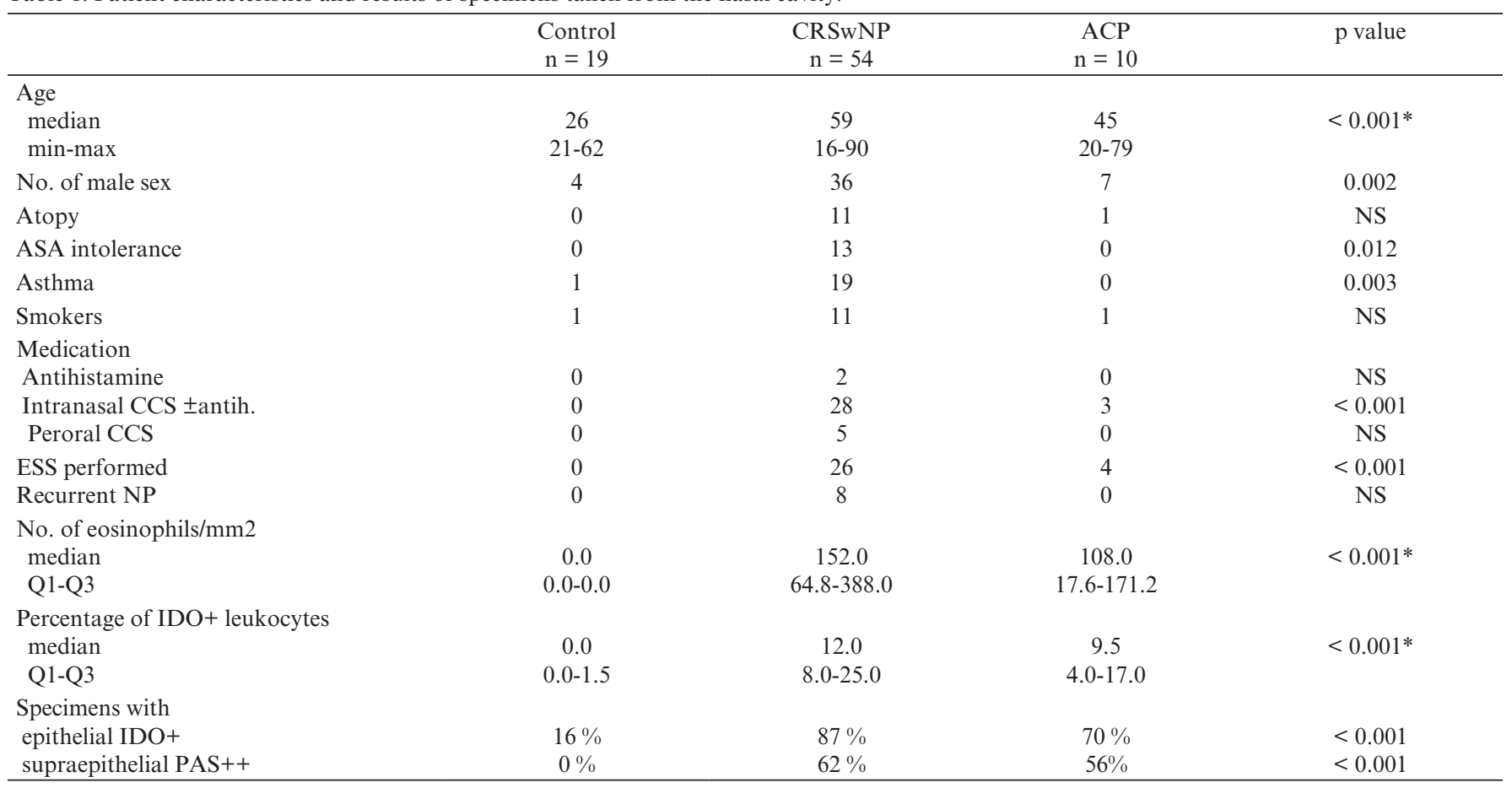

Control = mucosa from inferior turbinate from patient without inflammation of nasal mucosa and without sinonasal disease, CRSwNP $=$ chronic rhinosinusitis with nasal polyp, ASA = aspirin, ACP = antrochoanal polyp, CCS = corticosteroid, antih. = antihistamine, ESS = endoscpic sinus surgery, Q1- Q3 = 25 and 75 percentiles, respectively, IDO+ = positivity with $\mathrm{mAb}$ anti-indoleamine 2,3 dioxygenase, PAS $++=$ strong Periodic acid shiff -staining positivity, indicating much supraepithelial mucus. P-values by Fisher's exact test (discrete) or *Kruskal Wallis test (continuous). NS = not significant.

Table 2. Patient characteristics and results of specimens taken from the maxillary sinus.

\begin{tabular}{|c|c|c|c|c|}
\hline & $\begin{array}{c}\text { Control } \\
\mathrm{n}=12\end{array}$ & $\begin{array}{c}\text { CRSsNP } \\
\mathrm{n}=41\end{array}$ & $\begin{array}{c}\text { CRSwNP } \\
\mathrm{n}=14\end{array}$ & $\mathrm{p}$ value \\
\hline $\begin{array}{l}\text { Age } \\
\text { median } \\
\text { min-max }\end{array}$ & $\begin{array}{c}28 \\
19-56\end{array}$ & $\begin{array}{c}51 \\
16-75\end{array}$ & $\begin{array}{c}46 \\
16-76\end{array}$ & $0.003^{*}$ \\
\hline No. of male sex & 4 & 13 & 9 & NS \\
\hline Atopy & 5 & 25 & 8 & NS \\
\hline ASA intolerance & 0 & 0 & 7 & $<0.001$ \\
\hline Asthma & 0 & 13 & 8 & 0.003 \\
\hline Smokers & 3 & 9 & 2 & NS \\
\hline Medication & & & & \\
\hline Antihistamine & 0 & 4 & 2 & 0.067 \\
\hline Intranasal CCS \pm antih. & 0 & 16 & 7 & 0.001 \\
\hline Peroral CCS & 0 & 1 & 6 & $<0.001$ \\
\hline$\geq 1$ previous ESS & 0 & 10 & 4 & NS \\
\hline Hypertrophic polypoid mucosa & 0 & 11 & 14 & $<0.001$ \\
\hline $\begin{array}{l}\text { No. of eosinophils } / \mathrm{mm} 2 \\
\text { median } \\
\text { Q1-Q3 }\end{array}$ & $\begin{array}{c}80.0 \\
0.0-0.0\end{array}$ & $\begin{array}{c}82.7 \\
0.0-0.0\end{array}$ & $\begin{array}{c}72.6 \\
0.0-16.0\end{array}$ & NS * \\
\hline $\begin{array}{l}\text { Percentage of IDO+ leukocytes } \\
\text { median } \\
\text { Q1-Q3 }\end{array}$ & $\begin{array}{c}0.0 \\
24.0-160.0\end{array}$ & $\begin{array}{c}0.0 \\
48.0-261.3\end{array}$ & $\begin{array}{c}5.0 \\
31.6-198.3\end{array}$ & $<0.001^{*}$ \\
\hline $\begin{array}{l}\text { Specimens with } \\
\text { epithelial IDO+ }\end{array}$ & $42 \%$ & $51 \%$ & $71 \%$ & NS \\
\hline
\end{tabular}

Control = maxillary sinus mucosa from patient without chronic rhinosinusitis, CRSsNP = chronic rhinosinusitis without nasal polyps, CRSwNP = chronic rhinosinusitis with nasal polyps, ASA = aspirin, CCS = corticosteroid, antih. =antihistamine, ESS = endoscopic sinus surgery, Q1- Q3 = 25 and 75 percentiles, respectively,IDO+ = positivity with $\mathrm{mAb}$ anti-indoleamine 2,3 dioxygenase, P-values by Fisher's exact test (discrete) or * Kruskal Wallis test (continuous). NS = not significant. 
Table 3. Comparison of epithelial IDO+ in specimens from nasal cavity.

\begin{tabular}{lcccc}
\hline & OR & \multicolumn{2}{c}{ OR CI } & p-value \\
\cline { 3 - 4 } & & lower & upper & \\
\hline Control & ref & & & \\
CRSwNP & 35.8 & 10.5 & 122.6 & $<0.001$ \\
ACP & 12.4 & 2.7 & 57.8 & 0.007 \\
\hline
\end{tabular}

Non-adjusted odds ratio (OR), and lower and upper $90 \%$ confidence intervals (CI) for comparison of epithelial IDO+ in specimens from nasal cavity.

intranasal or peroral corticosteroids, or antihistamines, previous operations, and sex ( $p>0.05$ by Kruskal-Wallis test, data not shown). Age did not correlate with the percentage of IDO positive leukocytes ( $p>0.05$ by Spearman rank correlation test). There was no significant difference in the median percentage of IDO positive leukocytes in epithelial IDO+ and epithelial IDO- groups ( $p>0.05$ by Kruskal-Wallis test, data not shown).

Compared to control sinus mucosa, the number of mucosal eosinophils did not differ in CRSsNP or CRSwNP groups (p $>0.05$ by Kruskal-Wallis test, Table 2). The median number of eosinophils did not differ between epithelial IDO+ and epithelial IDO- groups ( $p>0.05$ by Kruskal-Wallis test, data not shown). The number of eosinophils $/ \mathrm{mm}^{2} \mathrm{did}$ not correlate with the percentage of IDO positive leukocytes ( $p>0.05$, by Spearman rank correlation test, data not shown).

\section{DISCUSSION}

We demonstrated that in the nasal cavity, epithelial IDO+ and the percentage of IDO+ leukocytes were enhanced in CRSwNP and ACP groups, when comparing to control inferior turbinate. In contrast, when observing the maxillary sinus mucosa, epithelial IDO+ did not differ significantly in CRSsNP or CRSwNP groups compared to control sinus mucosa, whereas the percentage of IDO positive leukocytes was enhanced in CRSwNP group.

In the nasal cavity, enhanced epithelial IDO+ and a higher percentage of IDO+ leukocytes during CRSwNP and ACP, but not in control nasal mucosa, might have a role in the pathogenesis of nasal polyp subgroups. By reducing locally the essential amino acid tryptophan, IDO might suppress the activation of inflammatory cells in the epithelial wall and mucosa of polyp tissue thus having putatively a role in decreasing inflammation and tissue damage. Because we did not observe the specimens from inflamed turbinate mucosa from CRSwNP patients, it remains unknown whether IDO+ is characteristic of polyp tissue or other inflammatory processes also.

We found eosinophils most abundantly during CRSwNP than in controls or ACP, which is in line with the previous observations ${ }^{(1,4)}$. Previously, it has been shown that the gene encoding the IDO genotype found from nasal polyp specimens might have a role in the genetic risk for ASA intolerant asthma that was observed from serum samples of different patients ${ }^{(31)}$. Here, ASA intolerance did not significantly affect the results. We demonstrated that, epithelial IDO+ and the high median percentage of IDO positive leukocytes associated significantly to each other and to a higher median number of eosinophils, which could support that both epithelial and leukocyte IDO might have an immunological role in polyp tissue especially if there is a predominance of eosinophilic inflammation. Whether the role of IDO is to promote or inhibit inflammation is not known ${ }^{(32)}$. The role of IDO in certain leukocytes might be related to the promotion of $\mathrm{T}$ cell tolerance; and in eosinophils, the function of IDO is either stimulatory or inhibitory depending on target cell and stimulus ${ }^{(24,33,34)}$. Further studies are required to analyse functionally active IDO in activated leukocyte subtypes and related cytokines during CRSwNP and CRSsNP.

In contrast to the nasal cavity, high numbers of control subjects had epithelial IDO+ in the maxillary sinus. Patient history factors were not associated with the IDO+ in sinus controls. The role of IDO+ in control sinus specimens could include the suppression of bacterial growth or the promotion of tolerance. Yet, more studies are required to bring evidence on this.

Epithelial IDO+ was found in all specimens of the sinus mucosa, it was not associated to CRS subgroups. In contrast, a high percentage of IDO+ leukocytes was significantly associated with CRSwNP. A study with IDO-/- mice did not reveal a role for IDO in antigen-induced immune tolerance in the airways but instead, IDO seemed to promote antigendriven Th2 responses via effects on lung dendritic cells ${ }^{(15)}$. This is in line with our finding that a high expression of IDO seems to be associated with CRSwNP group. In this study, all sinus specimens from patients with CRSwNP and about one quarter of specimens from CRSsNP group had microscopic signs of hypertrophic polypoid maxillary sinus mucosa (HP), the presence of which did not significantly affect the IDO expression. Thus, the putative role of histological HP on the pathogenesis of CRS requires further experiments.

The lower number of eosinophils in the sinus mucosa compared to NP, found in this study, is in line with previous observations ${ }^{(1)}$. In contrast, we did not find significant differences in the eosinophil numbers between different CRS subgroups, whereas others have shown CRSwNP to be associated with an elevated mucosal eosinophil count ${ }^{(3)}$. We demonstrated that epithelial IDO+ and the high median percentage of IDO+ leukocytes did not associate significantly to each other or to a higher median number of eosinophils in sinus mucosa. This controversy could possibly mean that IDO has at least partly a different immunological role in the sinus mucosa than in the nasal mucosa or polyp tissue. Still, further studies are warranted.

Others have shown that after in vivo aeroallergen exposure, serum IDO activity was increased in asymptomatic atopics 
compared with either symptomatic atopic or nonatopic individuals ${ }^{(35)}$. When exposing in vitro monocyte-derived dendritic cells with house dust mite Dermatophagoides pteronyssinus 1 , functionally active IDO decreased in cells from patients with house dust mite-sensitive asthma compared to nonatopic asthmatics ${ }^{(27)}$. Here we demonstrated that additional diagnosis of atopy did not statistically significantly affect the local IDO expression in CRS subgroups and ACP. In asthmatic patients, the baseline IDO activity in sputum has been shown to be significantly lower than control levels, but normal baseline activity was induced by using inhaled corticosteroids ${ }^{(36)}$. In this study, we observed that patient history of asthma or intranasal or peroral corticosteroid medication did not statistically significantly affect IDO expression in upper airways pathologies.

In conclusion, this study showed that in the nasal cavity, enhanced epithelial and leukocyte IDO are associated with both CRSwNP and ACP, but the association was strongest in CRSwNP. In the maxillary sinus, a higher percentage of IDO positive leukocyte is associated with CRSwNP but not with CRSsNP. The pathophysiological mechanisms underlying these findings require further studies.

\section{ACKNOWLEDGEMENTS}

This study was supported by research grants from the Competitive Research Fund of Pirkanmaa Hospital District, the Finnish Anti-Tuberculosis Association, the Finnish Society of Allergology and Immunology, the Finnish Society of Otorhinolaryngology, and the Tampere Tuberculosis Foundation. We thank Eini Eskola, Raija Hukkila, and Marja-Leena Koskinen for excellent technical assistance, and research nurse Marja-Leena Oksanen for excellent collaboration. We owe our gratitude to MD PhD Katriina Kostamo, and MD PhD Jari Suvilehto for valuable comments.

\section{REFERENCES}

1. Fokkens W, Lund V, Mullol J. European Position Paper on Rhinosinusitis and Nasal Polyps group. European position paper on rhinosinusitis and nasal polyps 2007. Rhinol. Suppl. 2007; 20: $1-136$.

2. Bousquet J, Khaltaev N, Cruz AA, et al. Allergic Rhinitis and its Impact on Asthma (ARIA) 2008 update (in collaboration with the World Health Organization, GA(2)LEN and AllerGen). Allergy. 2008; 63 Suppl 86: 8-160.

3. Bachert C, Van Bruaene N, Toskala E, et al. Important research questions in allergy and related diseases: 3-chronic rhinosinusitis and nasal polyposis - a GALEN study. Allergy. 2009; 64: 520-533.

4. Ebbens FA, Toppila-Salmi SK, Renkonen JA, et al. Endothelial L-selectin ligand expression in nasal polyps. Allergy. 2010; 65: 95-102.

5. Myller JP, Toppila-Salmi SK, Toppila EM, et al. Mucosal eosinophils and 1-selectin ligands are associated with invasive and noninvasive sinus surgery outcomes. Am J Rhinol Allergy. 2009; 23: $21-27$.

6. Jankowski R, Bouchoua F, Coffinet L, Vignaud JM. Clinical factors influencing the eosinophil infiltration of nasal polyps. Rhinology. 2002; 40: 173-178.

7. Kakoi H, Hiraide F. A histological study of formation and growth of nasal polyps. Acta Otolaryngol. 1987; 103: 137-144.

8. Van Zele T, Claeys S, Gevaert P, et al. Differentiation of chron- ic sinus diseases by measurement of inflammatory mediators. Allergy. 2006; 61: 1280-1289.

9. Van Bruaene N, Perez-Novo CA, Basinski TM, et al. T-cell regulation in chronic paranasal sinus disease. J Allergy Clin Immunol. 2008; 121: 1435-41, 1441.e1-3.

10. Maldonado M, Martinez A, Alobid I, Mullol J. The antrochoanal polyp. Rhinology. 2004; 42: 178-182.

11. Settipane GA, Chafee FH. Nasal polyps in asthma and rhinitis. A review of 6037 patients. J Allergy Clin Immunol. 1977; 59: 17-21.

12. Szczeklik A, Gryglewski RJ, Czerniawska-Mysik G. Clinical patterns of hypersensitivity to nonsteroidal anti-inflammatory drugs and their pathogenesis. J Allergy Clin Immunol. 1977; 60: 276-284.

13. Kowalski ML. Rhinosinusitis and nasal polyposis in aspirin sensitive and aspirin tolerant patients: are they different? Thorax 2000; 55 Suppl 2:S84-86.

14. Moffett JR, Namboodiri MA. Tryptophan and the immune response. Immunol Cell Biol. 2003; 81: 247-265.

15. Xu H, Oriss TB, Fei M, et al. Indoleamine 2,3-dioxygenase in lung dendritic cells promotes $\mathrm{Th} 2$ responses and allergic inflammation. Proc Natl Acad Sci USA. 2008; 105: 6690-6695.

16. Yoshida R, Urade Y, Tokuda M, Hayaishi O. Induction of indoleamine 2,3-dioxygenase in mouse lung during virus infection. Proc Natl Acad Sci USA. 1979; 76: 4084-4086.

17. Daubener W, MacKenzie CR. IFN-gamma activated indoleamine 2,3-dioxygenase activity in human cells is an antiparasitic and an antibacterial effector mechanism. Adv Exp Med Biol. 1999; 467: 517-524.

18. von Bubnoff D, Hanau D, Wenzel J, et al. Indoleamine 2,3-dioxygenase-expressing antigen-presenting cells and peripheral T-cell tolerance: another piece to the atopic puzzle? J Allergy Clin Immunol. 2003; 112: 854-860.

19. van der Marel AP, Samsom JN, Greuter M, et al. Blockade of IDO inhibits nasal tolerance induction. J Immunol. 2007; 179: 894-900.

20. Chen W, Liang X, Peterson AJ, Munn DH, Blazar BR. The indoleamine 2,3-dioxygenase pathway is essential for human plasmacytoid dendritic cell-induced adaptive $\mathrm{T}$ regulatory cell generation. J Immunol. 2008; 181: 5396-5404.

21. Taher YA, Piavaux BJ, Gras R, et al. Indoleamine 2,3-dioxygenase-dependent tryptophan metabolites contribute to tolerance induction during allergen immunotherapy in a mouse model. J Allergy Clin Immunol. 2008; 121: 983-991.e2.

22. Brenk M, Scheler M, Koch S, et al. Tryptophan deprivation induces inhibitory receptors ILT3 and ILT4 on dendritic cells favoring the induction of human CD4+CD25+ Foxp3+ T regulatory cells. J Immunol. 2009; 183: 145-154.

23. Xu H, Zhang GX, Ciric B, Rostami A. IDO: a double-edged sword for $\mathrm{T}(\mathrm{H}) 1 / \mathrm{T}(\mathrm{H}) 2$ regulation. Immunol Lett. 2008; 121: 1-6.

24. Grohmann U, Volpi C, Fallarino F, et al. Reverse signaling through GITR ligand enables dexamethasone to activate IDO in allergy. Nat Med. 2007; 13: 579-586.

25. Hayashi T, Beck L, Rossetto C, Gong X, Takikawa O, Takabayashi K, et al. Inhibition of experimental asthma by indoleamine 2,3-dioxygenase. J.Clin.Invest. 2004; 114: 270-279.

26. Gordon JR, Li F, Nayyar A, Xiang J, Zhang X. CD8 alpha+, but not CD8 alpha-, dendritic cells tolerize Th2 responses via contactdependent and -independent mechanisms, and reverse airway hyperresponsiveness, Th2, and eosinophil responses in a mouse model of asthma. J Immunol. 2005; 175: 1516-1522.

27. Maneechotesuwan K, Wamanuttajinda V, Kasetsinsombat K, et al. Der $\mathrm{p} 1$ suppresses indoleamine 2, 3-dioxygenase in dendritic cells from house dust mite-sensitive patients with asthma. J Allergy Clin Immunol. 2009; 123: 239-248.

28. Forsythe P, Inman MD, Bienenstock J. Oral treatment with live Lactobacillus reuteri inhibits the allergic airway response in mice. Am J Respir Crit Care Med. 2007; 175: 561-569.

29. Wolf AM, Wolf D, Rumpold H, et al. Overexpression of indoleamine 2,3-dioxygenase in human inflammatory bowel disease. Clin Immunol. 2004; 113: 47-55.

30. Torres MI, Lopez-Casado MA, Lorite P, Rios A. Tryptophan metabolism and indoleamine 2,3-dioxygenase expression in coeliac 
disease. Clin Exp Immunol. 2007; 148: 419-424.

31. Sekigawa T, Tajima A, Hasegawa T, et al. Gene-expression profiles in human nasal polyp tissues and identification of genetic susceptibility in aspirin-intolerant asthma. Clin Exp Allergy 2009; 39: 972-981.

32. Scheler M, Wenzel J, Tuting T, Takikawa O, Bieber T, von Bubnoff D. Indoleamine 2,3-dioxygenase (IDO): the antagonist of type I interferon-driven skin inflammation? Am J Pathol. 2007; 171: 1936-1943.

33. Odemuyiwa SO, Ghahary A, Li Y, et al. Cutting edge: human eosinophils regulate $\mathrm{T}$ cell subset selection through indoleamine 2,3-dioxygenase. J Immunol. 2004: 15; 173: 5909-5913.

34. Swanson KA, Zheng Y, Heidler KM, Mizobuchi T, Wilkes DS. CDllc+ cells modulate pulmonary immune responses by production of indoleamine 2,3-dioxygenase. Am J Respir Cell Mol Biol. 2004; 30: 311-318.

35. von Bubnoff D, Fimmers R, Bogdanow M, Matz H, Koch S, Bieber T. Asymptomatic atopy is associated with increased indoleamine 2,3-dioxygenase activity and interleukin-10 production during seasonal allergen exposure. Clin Exp Allergy 2004; 34: 1056-1063.
36. Maneechotesuwan K, Supawita S, Kasetsinsombat K, Wongkajornsilp A, Barnes PJ. Sputum indoleamine-2,3-dioxygenase activity is increased in asthmatic airways by using inhaled corticosteroids. J Allergy Clin Immunol. 2008; 121: 43-50.

Sanna (Toppila-)Salmi, MD, PhD

Helsinki University Central Hospital

Skin and Allergy Hospital

PO BOX 160

FI-00029 Hospital District of Helsinki and Uusimaa

Finland

Tel: +358-9-4711

Fax: +358-9-241 1227

E-mail: sanna.salmi@helsinki.fi 